\title{
CUIDADO DE ENFERMAGEM E EDUCAÇÃO EM SAÚDE COM PROFISSIONAIS DO SURF
}

Alacoque Lorenzini Erdmann ${ }^{1}$, Keyla Cristiane do Nascimento², Glaucia Krueger da Silva ${ }^{3}$, Sabrina Leitis Ramos ${ }^{3}$

\begin{abstract}
RESUMO: Sustentadas no referencial da Teoria das Necessidades Humanas Básicas de Horta, relata-se a vivência do trabalho de conclusão de curso de acadêmicas de Enfermagem no centro Desportivo e Cultural Araguá em Florianópolis/SC, num novo campo de atuação para os enfermeiros - a Enfermagem na atividade física e no esporte. Realizou-se o cuidado de Enfermagem e a educação em saúde aos atletas do surf, e às crianças da comunidade que participam das atividades que o Araguá oferece. Constatou-se, nesta prática assistencial, que a Enfermagem tem um papel indispensável no cuidado ao cliente, ao compor a equipe interdisciplinar dos profissionais que atuam nas atividades desportivas, e possibilita a ampliação de espaço com novas competências que seguramente contribui para o reconhecimento da Enfermagem como profissão.
\end{abstract}

PALAVRAS-CHAVE: Cuidados de Enfermagem; Enfermagem; Educação em Saúde.

\section{NURSING CARE AND HEALTH EDUCATION WITH SURF PROFESSIONALS}

ABSTRACT: Based upon Hortas's Basic Human Needs Theory, this study reports the graduation assignment performed by two nursing students at the Araguá Cultural and Athletic Center in Florianópolis, Santa Catarina, Brazil. This study examines a relatively new field of study for nurses - nursing in physical activity and sports. Nursing care and health education were provided to surf athletes, while the children of the local community participate in the activities offered at the Araguá Center. This practice showed that nursing has its indispensable place in client caring, taking part in the interdisciplinary team of sport professionals thus enabling the area to expand with new competencies that surely contribute for the recognition of the Nursing profession.

KEYWORDS: Nursing care; Nursing; Health education.

\section{ATENCIÓN DE ENFERMERIA Y EDUCACIÓN EN SALUD CON PROFESSIONALES DEL SURF}

RESUMEN: Basado en la Teoría de las Necesidades Humanas Básicas de Horta, el presente artículo relata las vivencias del trabajo de conclusión de curso de estudiantes de enfermería en el Centro Desportivo y Cultural Araguá em Florianópolis/SC en un nuevo campo para los enfermeros: la enfermería en la actividad física y en el deporte. En la práctica asistencial fue realizada la atención de enfermeria y la educación en salud a los atletas del surf y a los niños de la comunidad que participan de lss actividades que el local ofrece. En esta práctica, se ha constatado que la enfermería tiene un papel indispensable en el cuidado al paciente, al componer el equipo intersdisciplinario de los profesionales que trabajan en las actividades deportivas, posibilitando la ampliación del espacio con nuevas habilidades que seguramente contribuyen para el reconocimiento de la enfermería como profesión.

PALABRAS CLAVE: Atención de enfermería; Enfermería; Educación en Salud.

${ }^{1}$ Enfermeira. Doutora em Filosofia da Enfermagem. Professora Titular da Universidade Federal de Santa Catarina - UFSC. Orientadora do projeto de prática assistencial do Curso de Graduação em Enfermagem da UFSC.

${ }^{2}$ Enfermeira. Doutoranda em Enfermagem da UFSC.

${ }^{3}$ Discentes da $8^{\text {a }}$ fase do Curso de Graduação em Enfermagem da UFSC. 


\section{INTRODUÇÃO}

Historicamente, a Enfermagem tem mostrado à sociedade sua importância como profissão, em que o profissional tem como papel primordial cuidar, educar e, por muitas vezes, servir como único suporte para seu cliente. Todavia, com o passar das décadas, a Enfermagem veio e ainda vem se desenvolvendo e se especializando como uma ciência fundamental à sociedade e ao ser humano individualmente. Este progressivo e verdadeiro desenvolvimento é motivado pela constante luta por conquista de espaço e valorização da ciência de Enfermagem que os profissionais vivenciam diariamente na profissão. É nítido na rotina de qualquer enfermeiro, em qualquer que seja o campo de trabalho, a exigência de atitudes profissionais embasadas no seu conhecimento científico.

A Enfermagem se destaca como uma profissão versátil no que diz respeito ao campo de atuação. Como promotores de saúde, os enfermeiros são, dia após dia, requisitados nos mais diversos setores e grupos sociais e é de grande importância que estes profissionais estejam atentos à peculiar característica da profissão - a versatilidade de atuação - buscando incessantemente novos horizontes profissionais.

Com este intuito fomos buscar um novo campo de atuação para os enfermeiros em Florianópolis/SC - a enfermagem na atividade física e no esporte, por meio da realização do trabalho de conclusão de curso de duas alunas da graduação em Enfermagem da Universidade Federal de Santa Catarina (UFSC) no Centro Desportivo e Cultural Arágua, termo que significa exatamente ar + água.

A Enfermagem esportiva é uma área de trabalho que existe de fato, possui profissionais nela trabalhando, mas não é reconhecida, por contar com uma massa crítica ainda pequena para o desenvolvimento da área ${ }^{(1)}$.

$\mathrm{O}$ incentivo à prática desportiva vem sendo estimulada nos mais diversos níveis da população, principalmente por parte de instituições governamentais e não-governamentais, que buscam encaminhar crianças e adolescentes para a prática de atividades físicas, com a finalidade de promover saúde e otimizar esse movimento através de esportes, como futebol, vôlei, ginástica olímpica, tênis e surf.

Florianópolis é um dos locais mais privilegiados à prática do surf no Brasil. Esse esporte faz parte da cultura local e vem sendo praticado por pessoas de todas as idades. O Arágua, situado na Praia Mole -
Ilha de Florianópolis - SC, local escolhido para a execução desta prática assistencial, é uma instituição não-governamental que conta com o apoio financeiro de uma empresa de surf. É dotada de projetos ambientais, culturais, esportivos, de saúde e sociais, e vem incentivando a prática do surf não só aos atletas profissionais e amadores deste esporte, mas também às crianças da comunidade. Conta com apoio de profissionais do esporte e da saúde para o desenvolvimento das atividades, como médico, fisioterapeuta, psicólogo e nutricionista.

Assim como as demais disciplinas - medicina, psicologia, fisioterapia e nutrição - são áreas nas quais seus profissionais atuam possuindo fundamentação teórica para sua prática profissional no esporte, a Enfermagem tem seu espaço e papel importante na formação da equipe. Esse estudo veio confirmar a importância que o profissional enfermeiro tem na formação de uma equipe multiprofissional, com trabalho interdisciplinar que assiste a atletas e pessoas que praticam atividades físicas.

Para concretização deste estudo objetivou-se prestar cuidados de enfermagem segundo a teoria de Necessidades Humanas Básicas de Horta aos atletas profissionais e amadores do surf, e às crianças da comunidade que participam das atividades que o Arágua oferece, bem como acompanhar e participar da equipe multiprofissional que ali trabalha, buscando a interdisciplinaridade na assistência.

\section{REFERENCIAL TEÓRICO}

Para guiar essa prática assistencial optou-se pela Teoria das Necessidades Humanas Básicas (NHB) de Wanda de Aguiar Horta ${ }^{(2)}$ cujo foco é levar o ser humano à saúde e ao viver saudável, pelo atendimento de suas necessidades humanas básicas.

Horta fundamentou sua Teoria em uma abordagem Humanística e Empírica a partir da Teoria da Motivação Humana de Maslow, que organizou as NHB em cinco níveis: necessidades fisiológicas, de segurança, de amor, de estima e de auto-realização. Foi com esta percepção que Horta derivou seu modelo, o qual propõe uma metodologia para o processo de enfermagem sustentado na busca da satisfação de necessidades psicobiológicas, psicossociais e psicoespirituais ${ }^{(2)}$.

Para subsidiar a execução dessa prática assistencial, foram elaborados pressupostos que deram sustentação a esse estudo: Existe espaço para a Enfermagem atuar na área que envolve atividades 
físicas e esportes. O enfermeiro como um profissional que luta pela evolução de sua profissão, pode construir este espaço. A saúde é evidente em todos os espaços do contexto do viver do ser humano e, se necessário, o enfermeiro pode e deve intervir para a sua promoção. A prática de enfermagem centrada nas Necessidades Humanas Básicas promove o viver saudável. O ser humano que pratica atividades físicas e esportes, também pode ter suas Necessidades Humanas Básicas afetadas.

Os conceitos que nortearam este estudo foram: Enfermagem, ser humano, saúde, doença, ambiente, necessidades humanas básicas e cuidado de Enfermagem, embasados em Horta ${ }^{(2)}$. Para este estudo, sentiu-se necessidade de criar o conceito de Enfermagem na atividade física e no esporte.A Enfermagem na atividade física e no esporte é aqui entendida como a ciência e a arte de cuidar e ensinar o auto-cuidado para manter, promover e/ou recuperar a saúde, prestando assistência às necessidades humanas básicas daqueles que praticam algum tipo de atividade física e/ou esporte, seja de caráter profissional, ou não.

\section{BUSCANDO SUPORTE BIBLIOGRÁFICO}

Além de uma teoria que sirva de lastro para o estudo, é importante a revisão de literatura sobre aspectos que possam ajudar o esclarecimento ou aprofundamento da temática. Com essa intenção, serviram-nos como suporte teórico produções acerca da conceitualização de desportos e atleta relacionandoa à atuação da Enfermagem, a importância da atividade física para promover o viver e saúde/doença relacionados à atividade física.

\section{Desportos e Atletas}

De acordo com a Lei $\mathrm{n}^{\circ} 8.672 / 1993$ o desporto, como atividade predominantemente física e intelectual, pode ser reconhecido em qualquer das seguintes manifestações: desporto educacional, através dos sistemas de ensino e formas assistemáticas de educação, evitando-se a seletividade e a hipercompetitividade de seus praticantes [...]; desporto de participação, de modo voluntário, compreendendo as modalidades desportivas praticadas com a finalidade de contribuir para a integração dos praticantes na plenitude da vida social, na promoção da saúde e da educação e na preservação do meio ambiente e; o desporto de rendimento, praticado segundo normas e regras nacionais e internacionais, com a finalidade de obter resultados e integrar pessoas e comunidades do País e estas com outras nações ${ }^{(3)}$.

$\mathrm{O}$ grande impasse entre atividade física e esporte está justamente no fato de que enquanto na prática de uma atividade física a preocupação é a melhoria das condições de saúde e da qualidade de vida, no esporte a preocupação é atingir a produtividade máxima no mínimo tempo, e com o maior rendimento possível, tendo como conseqüência os prováveis prejuízos para a saúde em longo prazo $^{(4)}$.

Para o atleta, o esporte significa sua maior responsabilidade, que muitas vezes acontece em fases precoces da vida, enquanto que para o não-atleta praticar esportes é prazer e diversão, resultando na melhoria da qualidade de vida e no viver saudável ${ }^{(5)}$.

Em trabalho sobre Enfermagem no esporte Marques $^{(6)}$ a saúde desportiva pode ser considerada como a disciplina do futuro, devido à grande participação desta no campo da medicina preventiva.

$\mathrm{Na}$ atividade desportiva, não temos indivíduos doentes, e sim, pessoas saudáveis - atletas - que podem sofrer alguma lesão e conseqüentemente, dificultar seu desempenho nas atividades cotidianas. Esses atletas necessitam de atendimento na forma mais integral e humanística possível ${ }^{(1)}$. Cabe a nós, profissionais, estarmos atentos ao desenvolvimento desses atletas, identificar as necessidades de saúde e orientá-los para um acompanhamento e tratamento adequado, buscando atingir a otimização do desempenho físico, minimizando dores e, principalmente, favorecendo-os no sentido de proporcionar um retorno breve ao treinamento.

Exercícios físicos devem fazer parte dos hábitos saudáveis de todo ser humano, porém, a prática do esporte como profissão causa sofrimento, obriga o atleta a conviver com dor e lesões ocorridas na sua vida cotidiana.

Cabe aqui ressaltar que a prática assistencial realizada no Arágua foi dirigida aos atletas profissionais e amadores do surf.

\section{Saúde/Doença \& Atividade Física}

Atualmente, a busca pelo viver saudável vem sendo amplamente divulgada pela mídia, que tem induzido fortemente as pessoas à prática de atividades físicas e outros comportamentos em relação ao corpo.

Os principais benefícios à saúde advindos da prática de atividade física referem-se aos aspectos antropométricos, neuromusculares, metabólicos e psicológicos. Os efeitos metabólicos apontados por 
Matsudo e col. ${ }^{(7)}$ são: o aumento da potência aeróbica; o aumento da ventilação pulmonar; a melhora do perfil lipídico; a diminuição da pressão arterial; a melhora da sensibilidade à insulina e a diminuição da freqüência cardíaca em repouso. Em relação aos efeitos antropométricos e neuromusculares ocorrem a diminuição da gordura corporal, o incremento da força e da massa muscular, da densidade óssea e da flexibilidade.

$\mathrm{Na}$ dimensão psicológica, a atividade desportiva atua na melhoria da auto-estima, do autoconceito, da imagem corporal, das funções cognitivas e de socialização, na diminuição do estresse e da ansiedade e na diminuição do consumo de medicamentos ${ }^{(7)}$. A atividade física não é somente uma variável necessária para o equilíbrio, mas também um prazer de viver o próprio corpo.

A prática continuada de exercícios físicos, desde que em freqüência, duração e intensidade adequadas, produzem uma série de alterações no funcionamento do organismo como um todo ${ }^{(8)}$. Sabemos que a prática regular e adequada de exercícios físicos aumenta a capacidade da função cardiovascular. Aumentando-se a função cardiovascular significa dizer que o indivíduo adquire um sistema cardiovascular mais saudável e resistente a doenças coronarianas e outros problemas circulatórios, como: hiperlipidemias, hipertensão, entre outras.

Os indivíduos que praticam regularmente exercícios físicos por prazos superiores a seis meses apresentam inúmeras e conhecidas modificações em seu organismo, como diminuição da freqüência cardíaca em repouso e aceleração mais lenta durante o exercício, e redução dos níveis de gorduras sanguíneas, principalmente os triglicerídeos ${ }^{(8)}$.

A prática de atividade física também reduz a ansiedade e a depressão, regulariza o sono, melhora a auto-estima e o autoconceito, aumenta a autoconfiança além de reduzir a tensão muscular.

\section{Educação em saúde com crianças e adolescentes}

Padrões de vida saudáveis são adquiridos ainda na fase inicial da vida de cada ser humano. Portanto, nesta fase da vida é essencial o desenvolvimento de comportamentos saudáveis como a prática de atividade física. Nesta perspectiva, as ações educativas são os instrumentos de trabalho do profissional de saúde para o estabelecimento de um estilo de vida mais saudável.
As práticas educativas devem se fundamentar no uso de recursos lúdicos que simbolizem ou mesmo representem a temática, estimulando, assim, o estabelecimento de hábitos saudáveis em crianças e adolescentes. As atividades lúdicas de educação em saúde devem ser livres e incentivar a curiosidade e a criatividade de cada grupo etário em particular, apresentando-se de forma divertida e interessante.

Na definição de atividade lúdica enquadra-se qualquer atividade (jogos ou brincadeiras) que estimule a auto-expressão dos participantes, bem como leve ao relaxamento das tensões e, ainda, proporcione entretenimento e reconhecimento de si mesmo ${ }^{(9)}$.

Os jogos, brincadeiras e dinâmicas de grupo utilizados no processo de educação em saúde devem levar em consideração o desenvolvimento cognitivo de cada indivíduo. Vários autores mencionam os jogos educativos como uma forma de aprendizagem, e enfatizam, em especial, o uso no processo de aprendizagem de crianças e adolescentes ${ }^{(10)}$.

\section{METODOLOGIA}

Existem várias possibilidades de estudar os fenômenos e buscar as relações entre a teoria e a prática, as quais seguem procedimentos metodológicos para pesquisa e análise dos elementos estudados. Para subsidiar esta prática assistencial, optou-se pela Teoria de Enfermagem de Wanda de Aguiar Horta, respaldando a prática assistencial em seu modelo metodológico.

O Centro Desportivo \& Cultural Arágua foi o ambiente ou cenário escolhido para a realização deste trabalho de conclusão de curso. É uma instituição não governamental, dotada de projetos ambientais, culturais, esportivos, de saúde e sociais. Além dos surftreinos, alguns profissionais do esporte e da saúde fazem parte de atividades complementares para o desenvolvimento dos atletas, como o condicionamento físico, yoga, acompanhamento médico, psicológico, fisioterápico e nutricional.

O Arágua foi efetivado em 2002, já com a premissa de se tornar um centro de referência em treinamento para surf, integrando diversas atividades esportivas para um aperfeiçoamento da prática do esporte. Hoje, com esta complexa estrutura montada, o Arágua serve como ponto de treinamento e encontro, não somente para os atletas do surf, mas também de outros esportes de ação, como a natação, o windsurf, o kitesurf, o sandboard, o bodyboard, o triatlon, o 
wakeboard e vôo livre, com uma filosofia de preservação e harmonia com a natureza.

Em 2003, o Arágua passou a ser uma Organização de Sociedade Civil de Interesse Público OSCIP, que é pessoa jurídica de direito privado, sem fins lucrativos, formada para atender necessidades sociais.

Nesta experiência de prática assistencial buscou-se trabalhar com dois públicos-alvo distintos: crianças da comunidade que freqüentam a escolinha de $\operatorname{surf}$ (15 ao total), com idades entre cinco e dez anos, e atletas amadores e profissionais que participam das atividades e treinos de surf que o Arágua oferece.

No desenvolvimento deste trabalho, foram respeitados o Código de Ética Profissional e a Resolução 196/96, que levam em conta princípios éticos básicos que devem orientar qualquer estudo que envolva o ser humano, a saber: o respeito pela pessoa, a beneficência, a justiça, a fidelidade, a veracidade e a confidencialidade ${ }^{(11)}$. O estudo teve inicio após o consentimento da instituição devidamente oficializado pela Coordenação da disciplina de Prática Assistencial do Curso de Graduação em Enfermagem da Universidade Federal de Santa Catarina.

\section{VIVENCIANDO O CUIDADO NOS DESPORTOS}

O Arágua, como uma OSCIP, desenvolve trabalhos de cunho social sendo um deste a escolinha de surf para crianças entre cinco e dez anos de idade matriculadas na terceira série de uma Escola Pública Municipal. O grupo é formado por um total de quinze crianças. As aulas de surf são ministradas nas terças e quintas-feiras pela manhã por um professor de educação física, em uma iniciativa voluntária. Fazem parte da equipe, dois professores auxiliares (sem formação acadêmica) e dois acadêmicos de psicologia que desenvolvem atividades recreativas antes das aulas.

Como proposta de assistência de enfermagem a estas crianças, buscou-se desenvolver estratégias diversas que promovessem a saúde e prevenissem agravos e/ou complicações futuras. As estratégias traçadas para as 90 horas de prática assistencial com as crianças envolveram atividades educativas, acompanhamento do crescimento e desenvolvimento e atendimento de eventuais intercorrências.

O acompanhamento do crescimento e desenvolvimento cognitivo e neuropsicomotor das crianças, a obtenção de medidas antropométricas, o grau de nutrição e as imunizações foram quesitos avaliados através das consultas de enfermagem realizadas com as crianças acompanhadas pelos seus pais. Nessas consultas, verificávamos as carteiras de saúde e realizávamos o exame físico da criança, além de orientações aos pais sobre hábitos de vida saudável.

Nosso segundo foco de assistência foram os atletas amadores e profissionais que participam das atividades e treinos de surf. Com estes atletas, de faixa etária entre nove e vinte anos de idade, foram realizadas consultas de enfermagem, auxiliadas por um instrumento elaborado pelas autoras do trabalho.

Neste instrumento estavam contidas informações para identificação do atleta, histórico individual de saúde e antecedentes familiares, dentre outras informações relevantes para a avaliação da saúde do atleta. O instrumento foi formulado segundo a Teoria das Necessidades Humanas Básicas de Wanda de Aguiar Horta. A estrutura do instrumento, portanto, é baseada no modelo Weed de registros. Nas consultas eram realizadas anamnese, verificação de sinais vitais, registro e avaliação de medidas antropométricas, orientações sobre patologias e hábitos saudáveis, além de encaminhamentos para outros profissionais de saúde do próprio Arágua, concretizando, assim, o objetivo de realizar um trabalho interdisciplinar.

Com o transcorrer das consultas, foram diagnosticadas várias situações saúde/doença que demandaram orientações de enfermagem, como: distúrbios do sono e repouso, padrões de alimentação insatisfatórios, distúrbios gastrointestinais, doenças de pele, dentre outros agravantes de saúde.

Observou-se que os atletas do surf, em sua maioria, residem sozinhos ou com amigos, sem o cuidado diário de adultos responsáveis. Neste contexto, diversos jovens apresentam hábitos de vida inadequados para a idade (alimentação, sono e repouso prejudicados, ingestão de álcool, entre outros), trazendo risco para o desenvolvimento de algum agravo de saúde.

Através das consultas, observamos que apesar da existência de uma equipe multiprofissional e interdisciplinar de saúde, nem sempre os registros de saúde dos atletas eram compartilhados com toda a equipe. Portanto, sentimos a necessidade de elaborar um arquivo contendo todos os dados dos atletas, registrados por todos os profissionais da equipe.

Em concordância com o avanço tecnológico que temos vivenciado dia após dia, surgiu a idéia da construção de um banco de dados dos atletas. $\mathrm{Na}$ primeira consulta, eram preenchidos os dados iniciais: 
dados de identificação e histórico de saúde pessoal e familiar. A partir disso, cada profissional tem sua ficha inserida no programa, e a partir dela realiza sua consulta e alimenta o banco de dados. $\mathrm{O}$ acesso às informações contidas no banco de dados é permitido a todos os profissionais de saúde, tornando as informações sobre o atleta comum a todos, permitindo assim, uma assistência interdisciplinar e de qualidade.

Vale destacar que o instrumento de consulta de enfermagem está inserido no banco de dados da instituição, elaborado pelas autoras deste trabalho, sendo parte fundamental da avaliação interdisciplinar realizada pela equipe de saúde multiprofissional.

\section{PREVENINDO E PROMOVENDO O CUIDADO À SAÚDE}

A atividade educativa permeou toda a prática assistencial, ocorrendo paralelamente às consultas de enfermagem. Por estarmos trabalhando com grupos de faixa etária e atividades diferenciadas, optou-se por desenvolver educação em saúde com dois grupos de formas distintas: utilizando atividades lúdicas com as crianças e dinâmicas de grupo com os jovens.

As atividades educativas elaboradas de forma lúdica para as crianças tratavam questões de saúde, como: higiene corporal, preservação do meio ambiente, nutrição, cuidados com o sol, cuidados com o mar, doenças infecto-contagiosas (pediculose, escabiose, entre outros) e percepção do processo saúde-doença.

As dinâmicas de grupo trabalhadas abordavam conteúdos de saúde que estavam diretamente relacionados aos atletas do surf, como por exemplo: drogas, sexualidade e doenças sexualmente transmissíveis, infecções mais comuns no surfista (doenças respiratórias e de pele), saúde da mulher surfista, hábitos de vida saudável, entre outros.

Além das consultas de enfermagem e educação em saúde, as discentes acompanhavam as aulas de surf, os surftreinos e os campeonatos. Estavam sempre atentas para atendimento em eventuais intercorrências ou emergências que pudessem ocorrer durante as atividades de surf, como: lesões por trauma, lipotimia, ferimentos, hematomas, entorses, hipotermia, dentre outras. No decorrer da prática assistencial, observouse a carência de materiais de primeiros socorros na instituição para o atendimento a possíveis intercorrências durante as atividades, treinos e campeonatos de surf promovidos pelo Arágua. A partir disto, as autoras elaboraram uma listagem de materiais de atendimento imediato para a montagem de uma bolsa de primeiros socorros, que ficava à disposição da instituição.

\section{CONSIDERAÇÕES FINAIS}

Na realização dessa prática assistencial, observou-se a importância das atividades do enfermeiro para o sucesso do cuidado e educação em saúde com profissionais do surf. Constatou-se que a Enfermagem, nesta realidade, tem um papel indispensável na assistência direta ao cliente, ao compor a equipe interdisciplinar dos profissionais de saúde que atuam nas atividades desportivas.

Pela educação em saúde foi possível, principalmente, a promoção da saúde centrada em hábitos saudáveis de vida segundo a filosofia do grupo e da instituição. A interação ser humano e natureza como princípio para manter a melhor qualidade de vida e o viver mais saudável permeou toda a experiência de cuidado de enfermagem a estes sujeitos que praticam o surf.

$\mathrm{O}$ enfermeiro que trabalha nesta área pode ter sua assistência focada tanto nas pessoas que praticam atividades físicas na busca de uma melhor qualidade de vida, como nas pessoas que praticam atividades físicas na prevenção e/ou tratamento de algumas doenças. Além disso, o profissional pode assistir aos atletas que praticam toda a gama de esportes existente, direcionando sua assistência na prevenção e/ou tratamento de lesões.

É muito importante, neste contexto, que a prática assistencial da enfermagem seja diferenciada daquela prática tradicional e desenvolvida em serviços hospitalares ou ambulatoriais, permitindo que o centro da atenção seja a saúde, e não a doença. Assim, podese concluir que o novo campo de atuação, ainda pouco explorado pelos profissionais enfermeiros, está em constante crescimento, restando aos profissionais buscarem a qualificação adequada no que diz respeito à sua capacitação, para assumirem efetivamente essa área de atuação, que é a Enfermagem na atividade física e no esporte.

O referencial da Teoria das Necessidades Humanas Básicas de Horta utilizado para as consultas de enfermagem e demais atividades desta experiência de prática assistencial foi adequado, pois possibilitou segurança, domínio dos conhecimentos técnicos científicos aliados ao fazer segundo a estrutura do processo de enfermagem, propiciando um cuidado 
interativo e construtivo.

As atividades educativas constituíram importante método para estimular a compreensão e a aprendizagem das crianças e dos adolescentes. Os recursos utilizados apresentaram-se como instrumentos adequados para o desenvolvimento de práticas de educação em saúde com os usuários do Arágua.

A interdisciplinaridade entre os profissionais que cuidam de atletas foi vivenciada pela troca de conhecimentos centrados na filosofia do Arágua de promoção do viver mais saudável, integrado com a natureza, especialmente valorizando a relação ar e água para a saúde.

A Enfermagem, ao ampliar seu espaço de trabalho, tem nesse momento uma grande oportunidade de avançar, como disciplina profissional, na sua missão e, conseqüentemente, na sua autonomia e reconhecimento social.

\section{REFERÊNCIAS}

1 Kretly V. Enfermagem atuando no esporte. Nursing. 2004;71(7):10-1.

2 Horta WA. Processo de enfermagem. São Paulo: EPU, 1979.

3 Brasil, Senado Federal. Lei $n^{\circ} 8672$, de 6 de julho de 1993. Acesso em 14 de Março de 2005. Disponível: www.senado.gov.br

4 Canoletti B, Faro ACM. Exame físico do atleta: aplicação de um instrumento para a avaliação do enfermeiro [trabalho de conclusão de curso]. São Paulo (SP): Universidade de São Paulo; 2003.

5 Kretly V. O significado do esporte para o atleta: estudo com os (as) atletas do Centro Olímpico de Treinamento e Pesquisa [dissertação]. São Paulo (SP): Universidade de São Paulo; 2000.

6 Marques VH. Enfermagem no esporte: uma atividade possível [trabalho de conclusão de curso]. São Paulo (SP): Universidade de São Paulo; 2002.

7 Matsudo SM, Matsudo VKR. Evidências da importância da atividade física nas doenças cardiovasculares e na saúde. Diagn Trat. 2000;5(2):10-7.

8 Silva OJ, Silva TJC. Exercício e saúde: fatos e mitos. Florianópolis: UFSC, 1995.

9 Rabelo SE, Padilha MICS. A atividade lúdica no processo educativo ao cliente diabético adulto. Texto Contexto Enferm. 1998;3(7):106-17.

10 Kamii C, Deviries R. Jogos em grupo na educação infantil. São Paulo: Trajetória Cultural; 1991.

11 Ministério da Saúde(BR), Conselho Nacional de Saúde. Diretrizes e normas regulamentadoras de pesquisa em seres humanos. Resolução n ${ }^{\circ} 196$, de 10 de Outubro de 1996. Brasília; 1996. 